\title{
Geometric optics in the presence of axionlike particles in curved spacetime
}

\author{
Dominik J. Schwarz ${ }^{1},{ }^{1}$ Jishnu Goswami, ${ }^{1}$ and Aritra Basu $\circledast^{1,2}$ \\ ${ }^{1}$ Fakultät für Physik, Universität Bielefeld, Postfach 100131, 33501 Bielefeld, Germany \\ ${ }^{2}$ Thüringer Landessternwarte, Sternwarte 5, 07778 Tautenburg, Germany
}

(Received 31 March 2020; accepted 5 April 2021; published 27 April 2021)

\begin{abstract}
We present a concise derivation of geometric optics in the presence of axionic fields in a curved spacetime. Whenever light can be described via geometric optics (the eikonal approximation), the only difference to the situation without axionic field is the phenomenon of achromatic birefringence. Consequently, redshift of light and distance estimates based on propagating light rays, as well as shear and magnification due to gravitational lensing are not affected by the interaction of light with an axionic field.
\end{abstract}

DOI: 10.1103/PhysRevD.103.L081306

The study of light propagation in the presence of an axionlike field in an arbitrary spacetime geometry is of interest for constraints on axionic dark matter [1-6] and the search for the quantum chromodynamics (QCD) axion [7]. The latter would solve the strong $C P$ problem, but its coupling to photons, $g_{a \gamma}$, remains a free parameter [8].

Of late, ultralight axionlike particles are being probed through observations of distant astrophysical sources (e.g., [8-13] ). Therefore, photon propagation in non-Euclidean spacetime and their interaction with axionic fields needs to be established. Firstly, this is of interest for the search for axions and axionlike particles itself, but secondly, and perhaps more importantly, should axions or axionlike particles form the dark matter or even just exist as a subdominant but ubiquitous component of the Universe, we must ask if observational astrophysics and cosmology could be affected by an axionic medium. We demonstrate below that for all observations in which it is sufficient to use geometric optics, like for establishing the Hubble-Lemaître expansion of the Universe by means of observing standard candles or rulers, the presence of an ubiquitous axionic medium is irrelevant.

Typically, photon frequencies in astronomical observations and optical laboratory experiments are much larger than the typical frequency of axionic oscillations, which is $\nu_{a}=242\left[\mathrm{~m} / 10^{-21} \mathrm{eV}\right] \mathrm{nHz}$ for ultralight axionlike particles and $\nu_{a}=242[\mathrm{~m} / \mu \mathrm{eV}] \mathrm{MHz}$ for the QCD axion. Here, $m$ denotes the mass of the axionic field. If the corresponding photon wavelength being well below all

Published by the American Physical Society under the terms of the Creative Commons Attribution 4.0 International license. Further distribution of this work must maintain attribution to the author(s) and the published article's title, journal citation, and DOI. Funded by SCOAP ${ }^{3}$. typical length scales of the considered application, in particular the scale of gradients in the axionic field and in the structure of spacetime, the propagation of light can be described in the limit of geometric optics (the so-called eikonal approximation, [see e.g., [14] ]).

Here we assume that the frequency of the photons of interest is much larger than the corresponding mass of the axionic field, which is always satisfied for ultralight axionlike particles for typical astronomical observations performed in the electromagnetic spectrum above $\sim 10 \mathrm{MHz}$, but not necessarily true for the QCD axion. That excludes the interesting resonance phenomenon of the stimulated emission of photons from an axionic cloud [15-18].

In this communication we derive the most general equations of geometric optics in an arbitrarily curved spacetime and for an arbitrary configuration of an axionic field. Our result generalizes $[19,20]$ to arbitrary spacetimes; simplifies the derivation presented in [21]; and we demonstrate that the Etherington theorem [22] and the Sachs equations [23] remain unchanged for light propagation in axionic fields.

In this work, the signature of the metric tensor $g_{\mu \nu}$ is chosen to be $(-,+,+,+)$ and we use the HeavisideLorentz system with $\epsilon_{0}=\mu_{0}=c=\hbar=1$. The action

$$
S=\int d^{4} x \sqrt{-g} \mathcal{L}
$$

of the electromagnetic field coupled to an axionlike particle in a curved spacetime is given by the Lagrangian (our sign convention for the axion-photon coupling follows the one of [19])

$$
\mathcal{L}=-\frac{1}{4} F_{\mu \nu} F^{\mu \nu}-\frac{1}{2} \partial_{\mu} a \partial^{\mu} a+\frac{\mathrm{g}_{a \gamma}}{4} a F_{\mu \nu} \tilde{F}^{\mu \nu}-V
$$


where $F_{\mu \nu}$ denotes the electromagnetic field strength tensor and $\tilde{F}^{\mu \nu}=\epsilon^{\mu \nu \alpha \beta} F_{\alpha \beta} / 2$ its dual tensor. $a$ is an axionic field and $V \equiv V(a)$ its potential. Variation with respect to the gauge field $A_{\alpha}$ gives rise to the equation of motion (using $\nabla_{\mu} \sqrt{-g}=0$, where $\nabla_{\mu}$ denotes the covariant derivative),

$$
\nabla_{\mu} F^{\mu \alpha}-g_{a \gamma} \epsilon^{\mu \nu \rho \alpha} \partial_{\mu} A_{\nu} \partial_{\rho} a=0 .
$$

Making use of $\left[\nabla_{\mu}, \nabla_{\nu}\right] A^{\mu}=R_{\mu \nu} A^{\mu}$, with $R_{\mu \nu}$ denoting the Ricci tensor, and in Lorenz gauge, $\nabla_{\mu} A^{\mu}=0$, we obtain,

$$
\square A^{\alpha}-R_{\mu}^{\alpha} A^{\mu}-\mathrm{g}_{a \gamma} \epsilon^{\mu \nu \rho \alpha} \partial_{\mu} A_{\nu} \partial_{\rho} a=0 .
$$

Using the rules for exchanging covariant derivatives one can see that this equation and the Lorenz gauge are invariant under the residual gauge freedom $A^{\alpha} \rightarrow A^{\alpha}+\partial^{\alpha} f$ with $\square f=0$.

The equations of geometric optics follow from the ansatz $A^{\alpha} \equiv \operatorname{Re}\left[\mathcal{A}^{\alpha} \exp (\imath \psi)\right]$ and the assumption that large gradients in time and space are described by the real phase $\psi$, while all other changes are summarized by a complex, slowly evolving amplitude $\mathcal{A}^{\alpha}$. We define the wave vector

$$
k_{\mu} \equiv \partial_{\mu} \psi
$$

justified by the resulting equations of motion. An observer characterized by the spacetime velocity $u^{\mu}$ measures the circular frequency $\omega=-k_{\mu} u^{\mu}$. A direct consequence of the definition of the wave vector is

$$
\nabla_{\mu} k_{\nu}=\nabla_{\nu} k_{\mu}
$$

which reflects the property that the vector field $k_{\mu}$ is normal to the electromagnetic wave front, which defines a hypersurface of spacetime.

Inserting the ansatz into the equation of motion in Eq, (4) and the gauge condition, the terms of order $k^{2}$ and $k$ become

$$
\begin{gathered}
k_{\mu} k^{\mu}=0, \\
2 k^{\mu} \nabla_{\mu} \mathcal{A}^{\alpha}+\mathcal{A}^{\alpha} \nabla_{\mu} k^{\mu}-\mathrm{g}_{a \gamma} \epsilon^{\mu \nu \rho \alpha} k_{\mu} \mathcal{A}_{\nu} \partial_{\rho} a=0, \\
k_{\mu} \mathcal{A}^{\mu}=0,
\end{gathered}
$$

respectively [24]. The leading term in Eq. (7) is the null condition. Acting on it with a covariant derivative and using Eq. (6), we find the equivalent to the geodesic equation

$$
k^{\mu} \nabla_{\mu} k_{\alpha}=0
$$

Thus, in the limit of geometric optics and in the presence of a light axionic field (i.e., with $m \ll \omega$ ), light rays remain to be described by null geodesics in curved spacetime.
We further contract Eq. (8) with $\mathcal{A}_{\alpha}$, define $\mathcal{A}^{2}=\mathcal{A}_{\alpha} \mathcal{A}^{\alpha *}$, and obtain

$$
k^{\mu} \nabla_{\mu} \mathcal{A}+\frac{1}{2} \mathcal{A} \nabla_{\mu} k^{\mu}=0,
$$

which tells us how the light intensity $I=\omega^{2} \mathcal{A}^{2}$ (see below) changes along the light path and shows that the presence of a light axionic field does not affect the observed intensity of a light source.

Finally, let us introduce the normalized, spacelike (complex) polarization vector $\epsilon^{\mu}$, i.e., $\epsilon^{\mu} \epsilon_{\mu}^{*}=1$ and $\epsilon_{\mu} k^{\mu}=0$, and write $\mathcal{A}^{\alpha}=\mathcal{A} \epsilon^{\alpha}$. Returning to Eq. (8) and using Eq. (11) we find

$$
k^{\mu} \nabla_{\mu} \epsilon^{\alpha}-\frac{1}{2} g_{a \gamma} \epsilon^{\mu \nu \rho \alpha} k_{\mu} \epsilon_{\nu} \partial_{\rho} a=0 .
$$

This equation leads to the phenomenon of birefringence.

To see that most easily, let us introduce an orthonomal basis of vectors $\left\{u, e_{1}, e_{2}, n\right\}$, consisting of a timelike vector field $u^{\mu}$, describing a congruence of observers with $u_{\mu} u^{\mu}=-1$, a spacelike vector field $n^{\mu} \equiv\left(k^{\mu}-\omega u^{\mu}\right) / \omega$, pointing from the source to the family of observers, and $n^{\mu} n_{\mu}=1$, and the spacelike linear polarization basis $e_{i}^{\mu}$, $i=1,2$, which spans a screen normal to $n^{\mu}$. We can use the residual gauge freedom to set $\epsilon_{\mu} n^{\mu}=\epsilon_{\mu} u^{\mu}=0$. Then, $\epsilon_{\mu}=\varepsilon_{i} e_{\mu}^{i}$, with $e_{\mu}^{i} e_{j}^{\mu}=\delta_{j}^{i}$ and $\left|\varepsilon_{1}\right|^{2}+\left|\varepsilon_{2}\right|^{2}=1$. With $k^{\mu} \nabla_{\mu} e_{i}^{\alpha}=0$ (we parallel transport the basis vectors), we can now see that

$$
\left(\partial_{u}+\partial_{n}\right) \varepsilon^{i}-\frac{1}{2} \mathbf{g}_{a \gamma}\left[\left(\partial_{u}+\partial_{n}\right) a\right] \epsilon^{u j n i} \varepsilon_{j}=0 .
$$

This result is obtained from Eq. (12) by expressing the wave vector in terms of the base vectors and inserting a decomposition of unity in spatial and timelike components $\delta_{\beta}^{\alpha}=h_{\beta}^{\alpha}-u^{\alpha} u_{\beta}$, where $h_{\beta}^{\alpha}$ denotes the spatial projection operator, i.e., using $\delta_{\rho}^{\beta} \partial_{\beta} a=h_{\rho}^{\beta} \partial_{\beta} a-u_{\rho} \partial_{u} a$. The second term in Eq. (13) generates a rotation of the polarization components. Introducing the coefficients of circular polarization $\varepsilon_{L, R}=\left(\varepsilon_{1} \pm \imath \varepsilon_{2}\right) / \sqrt{2}$, respectively, we obtain two ordinary differential equations

$$
\varepsilon_{L, R}^{\prime} \pm \frac{l}{2} \mathrm{~g}_{a \gamma} a^{\prime} \varepsilon_{L, R}=0,
$$

where the prime denotes a derivative along the line of light propagation. The solution to that equation is

$$
\varepsilon_{L, R}\left(x_{\mathrm{o}}^{\mu}\right)=\exp ( \pm \imath \Delta) \varepsilon_{L, R}\left(x_{\mathrm{e}}^{\mu}\right),
$$

where $\Delta=\mathrm{g}_{a \gamma}\left[a\left(x_{\mathrm{e}}^{\mu}\right)-a\left(x_{\mathrm{o}}^{\mu}\right)\right] / 2$ is the rotation angle of the plane of linearly polarized light. Thus, in the limit of geometric optics, birefringence due to light axionic fields is 
achromatic, and depends on the coupling $g_{a \gamma}$ and the values of the axionic field at emission and observation. Moreover, the curvature of spacetime does not affect the birefringence angle $\Delta$, when expressed with respect to a parallel transported basis of linear polarization.

Chromatic and curvature effects show up when we go beyond the eikonal approximation, where we would actually return to a wave optics description of the system and no longer talk about light rays. Note that there are no nonlinear couplings in this limit. It was shown in [20,25] that beyond the limit of geometric optics, higher orders in the coupling lead to spectral distortions and refractive phenomena show up.

Finally, we can reformulate our results in the language of Stokes' parameters. In the limit of geometric optics and using the same gauge fixing as above, the electric field reads $E_{\alpha} \equiv F_{\alpha \beta} u^{\beta}=\operatorname{Re}\left[\omega \mathcal{A}_{\alpha} \exp (\nu \psi)\right]$. From that we find the four Stokes' parameters as follows:

$$
\begin{gathered}
I=\omega^{2} \mathcal{A}^{2}, \\
Q=I\left(\left|\varepsilon_{1}\right|^{2}-\left|\varepsilon_{2}\right|^{2}\right), \\
U=2 I \operatorname{Re}\left(\varepsilon_{1} \varepsilon_{2}^{*}\right), \\
V=-2 I \operatorname{Im}\left(\varepsilon_{1} \varepsilon_{2}^{*}\right),
\end{gathered}
$$

This implies that Stokes $I$ remains unchanged when photons propagate in an axionic field.

Let us give the example of a monochromatic and linearly polarized source. For such a source, we can write $\epsilon_{2}\left(x_{e}\right)=r \epsilon_{1}\left(x_{e}\right)$, with $r$ real, which gives

$$
\begin{aligned}
& Q=\frac{I}{1+r^{2}}\left[\left(1-r^{2}\right) \cos (2 \Delta)-2 r \sin (2 \Delta)\right], \\
& U=\frac{I}{1+r^{2}}\left[2 r \cos (2 \Delta)+\left(1-r^{2}\right) \sin (2 \Delta)\right] .
\end{aligned}
$$

Here, $Q^{2}+U^{2}=I^{2}$ and $V=0$.

One could imagine to set up a table top experiment based on finding birefringence of a polarized laser beam in vacuum, which could probe the mass range of the QCD axion. In astrophysics the situation of the simple example is found in the case of the face-on observation of protoplanetary discs [9]. Due to the galactic distance scale, ultralight axions can be probed in that case. For other sources with continuous emission, the relations for the Stokes' parameters may be more complicated, but the underlying principle of birefringence remains the same.

We have shown that axionic dark matter neither affects the geodesics of light, nor its intensity and therefore the Etherington theorem [22], the relation between angular and luminosity distance, and the Sachs equations [23], which describe the propagation of light bundles in curved spacetimes, also hold true in the presence of axion-photon interaction and in the limit of geometric optics with $m \ll \omega$. Thus, cosmic distance estimation, based on parallaxes, standard candles or standard rulers are not affected. Also the axion-photon interaction cannot change the redshift of a source. The only effect axionic fields have on light is the phenomenon of achromatic birefringence. This is in addition to the usual chromatic birefringence introduced by magnetized plasma [26,27].

Our result further implies, that the presence of axionic dark matter does not modify the conclusions on gravitational lensing as long as shape and magnification are concerned. This allows one to use strong gravitational lensing of linearly polarized extragalactic sources as a robust probe of ultralight axionlike particles, as demonstrated recently [13].

As a further generalization, one could invoke the full magnetized plasma interactions. A first step in this direction has been done for a cold nonmagnetized plasma in Minkowski spacetime [25]. This investigation showed that subdominant additional effects of the order of the plasma frequency squared, times the axion-photon coupling, are expected. Thus, the achromatic nature of axionic birefringence might provide a smoking gun in the search for ultralight axionic dark matter on cosmological and astrophysical length and time scales.

We thank Yuko Urakawa for very helpful discussions and comments, and, Walter Pfeiffer for discussions regarding future implications on laboratory experiments. We acknowledge support by the Deutsche Forschungsgemeinschaft (DFG, German Research Foundation) through the CRC-TR 211 "Strong-interaction matter under extreme conditions" Project No. 315477589 TRR 211 and by the German Federal Ministry of Education and Research (BMBF) under Grant No. 05A17PB1 (Verbundprojekt D-MeerKAT).
[1] J. Ipser and P. Sikivie, Phys. Rev. Lett. 50, 925 (1983).

[2] F. W. Stecker and Q. Shafi, Phys. Rev. Lett. 50, 928 (1983).

[3] J. Preskill, M. B. Wise, and F. Wilczek, Phys. Lett. 120B, 127 (1983).
[4] L. F. Abbott and P. Sikivie, Phys. Lett. 120B, 133 (1983).

[5] M. Dine and W. Fischler, Phys. Lett. 120B, 137 (1983).

[6] P. Sikivie and Q. Yang, Phys. Rev. Lett. 103, 111301 (2009). 
[7] J. E. Kim and G. Carosi, Rev. Mod. Phys. 82, 557 (2010); 91, 049902(E) (2019).

[8] G. Sigl and P. Trivedi, arXiv:1811.07873.

[9] T. Fujita, R. Tazaki, and K. Toma, Phys. Rev. Lett. 122, 191101 (2019).

[10] M. M. Ivanov, Y. Y. Kovalev, M. L. Lister, A. G. Panin, A. B. Pushkarev, T. Savolainen, and S. V. Troitsky, J. Cosmol. Astropart. Phys. 02 (2019) 059.

[11] S. Chigusa, T. Moroi, and K. Nakayama, Phys. Lett. B 803, 135288 (2020).

[12] Y. Chen, J. Shu, X. Xue, Q. Yuan, and Y. Zhao, Phys. Rev. Lett. 124, 061102 (2020).

[13] A. Basu, J. Goswami, D. J. Schwarz, and Y. Urakawa, arXiv:2007.01440.

[14] L. D. Landau and E. M. Lifshitz, Klassische Feldtheorie, Lehrbuch der Theoretischen Physik, Band 2 (AkademieVerlag, Berlin, 1987).

[15] I. I. Tkachev, Phys. Lett. B 191, 41 (1987).

[16] A. Caputo, M. Regis, M. Taoso, and S. J. Witte, J. Cosmol. Astropart. Phys. 03 (2019) 027.
[17] A. Arza and P. Sikivie, Phys. Rev. Lett. 123, 131804 (2019).

[18] D. G. Levkov, A. G. Panin, and I. I. Tkachev, Phys. Rev. D 102, 023501 (2020).

[19] D. Harari and P. Sikivie, Phys. Lett. B 289, 67 (1992).

[20] D. Blas, A. Caputo, M. M. Ivanov, and L. Sberna, Phys. Dark Universe 27, 100428 (2020).

[21] M. A. Fedderke, P. W. Graham, and S. Rajendran, Phys. Rev. D 100, 015040 (2019).

[22] I. M. H. Etherington, Philos. Mag. 15, 761 (1933).

[23] R. Sachs, Proc. R. Soc. Ser. A 264, 309 (1961).

[24] Note that for $\omega \sim m$, the last term in Eq. (8) would actually be of order $k^{2}$; therefore, our approximation does not capture the resonant case. For flat spacetimes, a similar ansatz has been worked out in Ref. [18].

[25] J. I. McDonald and L. B. Ventura, Phys. Rev. D 101, 123503 (2020).

[26] C. L. Carilli and G. B. Taylor, Annu. Rev. Astron. Astrophys. 40, 319 (2002).

[27] T. Akahori and D. Ryu, Astrophys. J. 723, 476 (2010). 